\title{
Where is the Ethics in Aesthetic Dentistry?
}

\author{
David L Hussey, Consultant in Restorative Dentistry, Queen's University of Belfast
}

\author{
Collins Concise English Dictionary (3rd Edition) provides the following definitions: Ethic - moral principle or set of \\ moral values held by an individual or group. Aesthetic - relating to pure beauty rather than to other \\ considerations. Cosmetic - having no other function than to beautify.
}

The term aesthetic dentistry has been used to refer to the application of tooth coloured restorations in either the anterior or posterior situation where non-tooth coloured alternatives are available. Unlike the dictionary definition there is a level of function that must be considered during the replacement of diseased tooth substance.

Cosmetic dentistry differs in that it is the application of restorative techniques purely to improve the appearance whilst not necessarily improving function. As a profession we have to marry these facilities with our ethical duty to provide patients

\section{The dental profession have to ensure that we are able to justify our treatment plans based on clinical need. . . allowing patients to have an acceptable level of input.}

with treatments which address their dental problems but not at the cost of the unnecessary destruction of sound tooth substance. I was spurred to address this issue following my attendance at several meetings in North America where presenters had the opportunity to demonstrate their undoubted skills in the provision of tooth coloured restorations. Unfortunately the cases used to illustrate these skills did not always reflect a high level of case selection.

In everyday practice dentists are faced with many decision-making situations, to

\section{CORRECTION}

Professor Ruth Freeman from Queen's University, Belfast, was the author of the conference leader on 'Oral Health Promotion Experience in Northern Ireland (BDJ 2002; 192: 175) and not Brian Mullally. extract or root treat, to treat or refer, NHS or private. Fortunately most of these decisions are based on clinical experience backed up with some level of evidence base. In many cases these decisions are purely clinical with no ethical component. There can be no argument that when dentists have the opportunity to restore teeth with materials that are clinically efficient and tooth coloured then the aesthetic material can be used at no detriment to the patient. The problem arises when there are non-aesthetic materials that are known to be superior in clinical function to tooth

coloured materials. This is further complicated when there is little difference between the performance of the materials involved.

Many practitioners have been faced with patients who have concerns over amalgam fillings despite such restorations being clinically sound and performing well. The problem of perceived allergy or sensitivity is a common cause for such patients seeking referral to Consultants in Restorative Dentistry. The moral dilemma is not made any easier when patients have sought the opinion of non-dental health professionals who have declared with conviction that the patient must have all their amalgam fillings removed to improve their general health status. In a recent assessment of this problem Dodes (2001) has reviewed the literature and it is clearly not a viable position to offer this treatment in the hope of improving general health problems. When the source of the opinion is a "proper doctor" it makes the dentist's situation unenviable. The dental profession have to ensure that we are able to justify our treatment plans based on clinical need but also allowing patients to have an acceptable level of input. This may include a choice of materials to be used based on cost, appearance and evidence of efficacy.

In what I have termed cosmetic dentistry, restorations such as veneers or crowns make a valuable contribution to the treatment of patients with severely discoloured or malformed teeth. However, such treatments have also been placed in an attempt to overcome minor irregularities that could be amenable to more traditional approaches; such as orthodontics which has proven successful in adult as well as adolescent cases.

Where the treatment is non-invasive and effective, such as bleaching, there should be no argument against its use as long as relevant health concerns have been addressed. There is really no moral question to be faced except perhaps the cost which is charged and I have no strong opinion in this matter, as there is such a range of practice expenses to be covered by the practitioner.

So, where is the ethics in aesthetic dentistry? I hope it is alive and well and thriving in our decision-making processes.

1. Dodes J E. The amalgam controversy. An evidence based analysis. JADA 2001; 132: 348-355.

The 2002 British and Irish Dental Associations's Annual Conference is being held at Belfast Waterfront Hall between 2-4 May 2002. Contact BDA Events office for further information.

Tel: +44 (020) 7563 4590; Fax: +44 (020) 75634591.

Website: www.bda-events.org.uk 